\title{
ESTUDOS PARA AUTOMAÇÃO DO PROJETO A ALVENARIA RACIONALIZADA EM PLATAFORMAS BIM
}

\author{
Levi Teixeira Pinheirol, Daniel Ribeiro Cardo ${ }^{2}$, Alexandre Araújo Bertini ${ }^{3}$, \\ 1 Professor efetivo, Instituto Federal do Ceará, CEP, Crateús, Brasil. \\ 2 Professor efetivo, Universidade Federal do Ceará, CEP , Fortaleza, Brasil.. \\ 3 Professor efetivo, Universidade Federal do Ceará, CEP, Fortaleza, Brasil.
}

*E-mail: levi.teixeira@terrambiente.com.br

\section{RESUMO}

A presente pesquisa trata da investigação de caminhos para automação do projeto de alvenaria em sistemas computacionais. Logo, é proposto o desenvolvimento diagramas aplicáveis em plataformas BIM, que permitam a geração automática da paginação de alvenarias racionalizadas em dois tipos de amarração: 1/2 e 1/3. A estratégia de pesquisa utilizada foi o constructive reasearch, tendo como base para o método de notação do comportamento do objeto paramétrico, denominado originalmente de building object behavior (BOB) desenvolvido no período de 2001 a 2004 por pesquisadores norte-americanos, no caso, Chang Lee, Rafael Sacks e Charles Eastman. A pesquisa tem sua importância por contribuir para o desenvolvimento das plataformas BIM, em específico na etapa de projeto, otimizando na produtividade e eficiência. Ao fim da pesquisa foi possível gerar o desenvolvimento dos diagramas e ainda compreender o grau de convergências e divergências existentes entre os mesmos no quesito de diagramas computacionais.

Palavras-chave: Automação do design, BIM, modelagem paramétrica, alvenaria racionalizada.

\section{Introdução}

Nas últimas duas décadas a indústria da construção civil do Brasil tem apresentado um crescimento exponencial em termos de produção [1]. Porém, a mesma ainda possui graves problemas no método e na forma de produção, por ainda depender de um processo construtivo muito artesanal, que apresenta falhas no fluxo de informações. O mesmo problema abrange todo ciclo de vida do edifício, desde a etapa de planejamento, que inclui projeto e orçamento até o processo executivo em si [2]. [3] aponta a industrialização e a racionalização como meios de otimizar o processo construtivo, através do Projeto para Produção de Vedações Verticais em Alvenaria (PVVA). Já a falha no fluxo de informações pode ser solucionada com a adoção do sistema BIM, Building Information Modeling [4].

O PPVA foi desenvolvido no mercado brasileiro em meados da década de 1980 [5] e visa otimizar o processo de projeto da alvenaria, auxiliando sua compatibilização com os diversos subsistemas que interagem com ele, como instalações e estruturas. O BIM tem como base as tecnologias de parametrização [6] que, por meio de objetos paramétricos, permitem o gerenciamento e ordenamento de dados aplicados às fases de ciclo de vida de um edifício [7]. Assim, a partir dessas ferramentas o sistema permite com facilidade identificar a incompatibilidades de projetos, extrair quantitativos automáticos e gerar instanciações do modelo 2D e 3D [8]. Devido à complexidade de cada projeto aliada, as plataformas BIM não oferecem objetos paramétricos suficientes para $\mathrm{O}$ desenvolvimento dos mesmos. Logo, a maioria dos programas BIM permite sua customização [9]. Os níveis de customização de software BIM são: (i) script, que é a criação de novos objetos paramétricos baseado em objetos existentes; (ii) plug in que é a criação de novas funcionalidades não existentes e; (iii) aplicativo, que é a criação de uma nova plataforma [10]. Além dos níveis de customização, observam-se as limitações de automação dos objetos paramétricos, que se baseiam nos métodos de mudança utilizados e nas rotinas automáticas [9].

A possiblidade de automação do design possui limitações de acordo com o método utilizado. Assim, como métodos de automação do design têm-se: a adaptação paramétrica; a substituição de partes e a adaptação topológica. No caso da adaptação paramétrica, a mudança é regida por algoritmos, onde estímulos externos ou internos sobre um objeto podem induzir a mudança. Até mesmo sem estímulos, podem ser gerados objetos automáticos, como é o caso da automação do layout, que será apresentado neste trabalho. No método de substituição de partes, os estímulos externos podem gerar automaticamente a substituição de componentes de um objeto. Porém, para um 
objeto atingir esse comportamento são necessárias regras sofisticadas. Como exemplo, tem-se a inserção de uma carga sobre uma determinada viga que, ao invés de ter suas dimensões geométricas modificadas, substitui a sua quantidade de ferragens automaticamente. As adaptações topológicas são mudanças que não podem ser automatizadas, devido às inúmeras possibilidades formais que o objeto pode atingir na presença de determinados estímulos. $\mathrm{O}$ que exigiria da plataforma computacional algoritmos muito complexos. Logo, tais adaptações são desenvolvidas por rotinas manuais [9].

Dentro dos métodos de mudança observamos rotinas automáticas do tipo: projeto de peças e conexões, a decomposição edílica e a geração automática do layout. $\mathrm{O}$ projeto de peças e conexões ocorre através de estímulos externos, como cargas estruturais sobre uma peça e suas conexões internas, onde a mesma se modifica em termos dimensionais, sendo a sua quantidade de conexões refeita. A decomposição edílica em camadas agrupadas ocorre com a inserção de estímulos externos, como uma carga em um modelo de edifício, sendo toda sua estrutura remodelada automaticamente, gerando novas dimensões e a redistribuição de conexões para cada peça envolvida no modelo. Todo o processo gera uma reação em cadeia. A geração automática do layout consiste na capacidade de distribuição automática de objetos no espaço, como a disposição de pilares, vigas, lajes, parede e, até de edifícios inteiros por meio da descrição de parâmetros [9].

Com demandas apresentadas pelos usuários, aliadas as possibilidades de a customização das plataformas, a customização de plataformas BIM vem se tornando um nicho de pesquisas acadêmicas. Como se observa no trabalho de [11] há várias possibilidades de customizações dos softwares. Porém, esta pesquisa está focada na área de automação de design aplicada a plataformas BIM. Assim, na esfera internacional, observa-se que existem pesquisas geradas a partir de um convênio entre empresas norte-americanas de concreto armado e universidades. Um exemplo de convênio ocorre entre uma universidade norte-americana (Georgia Institute) e outra israelita (Israel Institute of Technology) denominado Precast Concrete Software Consortium (PCSC), desenvolvido no período de 2001 a 2004. A pesquisa gerou o desenvolvimento de vários objetos paramétricos ligados ao sistema construtivo em concreto armado, aplicados a plataformas BIM e de métodos de automação do design: Building Object Behavior (BOB) e o Georgia Tech Process to Product Modeling (GTPPM) [12]. Dentre os pesquisadores envolvidos destacam-se Rafael Sacks, Charles Eastman e Chang Lee, sendo os dois primeiros também responsáveis pelo desenvolvimento do livro BIM Hand Book, publicado em 2008, documento que compila estado da arte do BIM e se apresenta como um guia para sua implementação [13]. Além destes, temos o trabalho de [14] pesquisador do Georgia Institute que aplicou o método BOB na automação de projetos de alvenaria estrutural, onde foram geradas regras de automação para parede isolada como: a inserção de paginações sobre paredes curvas. Foram deixadas, no entanto, as seguintes lacunas: o desenvolvimento de novas funções de automação, que gerem paginação automática da parede quando houver a da mesma interface com outros subsistemas.

Em nível nacional destacam-se trabalhos de customização de softwares BIM em diversas áreas [11], porém somente poucos se destacam na área de automação de design como no caso [15], [3], além de [16], que foram desenvolvidos de forma independente. Os trabalhos são consequência de dissertações de mestrado que consistem em estudos sobre automação de sistemas construtivos modulares, tendo como base metodológica a modelagem paramétrica e a gramática da forma.

Romcy [16] desenvolveu seu trabalho, tendo como resultados o desenvolvimento de um método de automação para sistemas construtivos de alvenarias modulares, com aplicação em paredes de tijolos cerâmicos na amarração $1 / 3$ e painéis de $d r y$ wall. Porém, o mesmo trabalho não possui um estudo da automação da alvenaria em interface com outros subsistemas (lajes, instalações e esquadrias). Monteiro et al [3] desenvolveu uma linguagem visual (Masonry Modulation Language) para representação do projeto de alvenaria em plataformas BIM, utilizando-se da metodologia de modelagem generativa e gramática da forma, com o intuito controlar o desempenho da plataforma. Ayres et al [15] apresentam uma discussão sobre os requisitos necessários a uma ferramenta $\mathrm{CAD} / \mathrm{BIM}$ direcionada para automatização de documentos de projeto de alvenaria de blocos de concreto, mas não geram de fato uma automação.

Ao analisarmos os trabalhos anteriores, observa-se o uso dos seguintes métodos para automação do design: gramática da forma e modelagem paramétrica, dentro da qual encontram-se o método BOB e GTPMM. A gramática da forma é um sistema de geração de formas para a pintura, a escultura e arquitetura, que por meio de regras de composição e se enquadra na área do conhecimento denominado design computing ou computational design. O termo computing não quer dizer, necessariamente, aplicação direta em aplicativos computacionais, mas sim a qualquer tipo de processamento de informações [17]. Por outro lado, a modelagem paramétrica permite uma melhor ligação entre funcionalidade do projeto e da geometria [14], pois é a base para o desenvolvimento das tecnologias de parametrização [18] Dentre as vantagens apresentadas por esta ferramenta, destacamse a facilidade de incorporação de conhecimento técnico sobre o comportamento do material, a fabricação e o processo construtivo nos estágios iniciais, melhorando a compreensão arquitetônica para implicações de projeto e permitindo sucesso nas inovações tecnológicas [18].

O método BOB pode ser compreendido como uma escrita abreviada e um protocolo para descrição das definições e comportamento de objetos paramétricos em um formato reutilizável e compartilhável [12]. O mesmo se desenvolveu a partir dos seguintes comportamentos geométricos individuais dos objetos: fixação, rotação, translação e remodelação e relações geométricas entre os objetos. A fixação faz referência à manutenção do objeto fixo em determinada situação. A rotação se refere às possibilidades de movimentação do objeto sobre ele 
mesmo a partir de um determinado eixo. A translação verifica possibilidade de deslocamento do objeto sobre o espaço. Já a remodelação se refere ao comportamento dimensional do objeto, isto é, as transformações de sua forma. Em termos de relações que podem ocorrer entre os objetos têm-se/estão às relações de alinhamento, igual espaçamento horizontal e restrição normal [14].

O GTPMM é, antes de tudo, um método de modelagem de produtos focado no processo de produção, que consiste em capturar informações precisas de cada etapa de produção do produto, como etapa de projeto, subcontratação e fornecimento de peças. O método é desenvolvido em três etapas. A primeira é a fase de coleta de requisitos onde são coletados os requisitos de modelagem, utilizando-se de métodos de modelagem da informação como: Fluxograma, Unified Modeling Language (UML), Design for Six Sigma (DFSS) e o Integrated Computer Aided Manufacturing Definition (IDEF0). Depois temos a fase lógica, onde os requisitos organizados na fase anterior são traduzidos para uma linguagem lógica do tipo EXPRESS-G e EXPRESS. A última fase é a própria implementação do modelo em uma plataforma, como o ArchiCad, Revit, dentre outras plataformas BIM [12].

Ao observamos os trabalhos anteriores, suas lacunas e as metodologias empregadas, propomos como objetivo deste trabalho o desenvolvimento de diagramas aplicáveis em plataformas BIM, que permitam a geração automática da paginação de alvenarias racionalizadas em dois tipos de amarração $1 / 2$ e $1 / 3$. O desenvolvimento dos diagramas tem como base o método BOB de notação do objeto paramétrico, que se enquadra na estratégia de pesquisa constructive reasearch, que diferentemente da ciência convencional, não propõe a descoberta de algo novo e sim a sua criação. No caso, um artefato que visa resolver problemas do mundo real, cuja contribuição teórica ocorre a partir de sua aplicação. Dentre artefatos que podem ser produzidos tem-se: modelos, diagramas, planos, produtos comerciais e design de sistemas de informação [19]. No caso dessa pesquisa trabalhamos com o desenvolvimento de diagramas.

\section{Parte experimental ou metodologia}

A pesquisa utilizará o método de notação e descrição BOB desenvolvido por Lee, Sacks e Eastman [12], que terá como suporte científico a estratégia de pesquisa design science. Esta estratégia, diferentemente da ciência convencional, não se propõe a descobrir nada novo e sim, produzir algo novo, isto é, um artefato.

O design science busca a resolução de problemas ou melhoria de desempenhos, que podem ocorrer por dois caminhos: melhoria da qualidade do produto e melhoria da qualidade do processo de produção [20].E para o desenvolvimento do mesmo utilizaremos o método BOB com suas devidas adaptações, devido a ausência de um programador.
O método $\mathrm{BOB}$ original é desenvolvido em quatro etapas: fase de elucidação fase de design, tradução e validação.

A fase de design ocorre quando são verificados as restrições e parâmetros do sistema construtivo, além de serem identificados os principais padrões de comportamentos. A fase de validação, onde os padrões identificados são submetidos a testes dentro de uma plataforma $A r c h i C A D$ 18, que permitem a comprovação do seu comportamento, por meio de simulações em script. Por fim a fase de tradução, onde os comportamentos são transcritos para diagramas de atividades em linguagem UML.

\section{Resultados e discussões}

\subsection{Fase de elucidação}

Nesta fase, o comportamento paramétrico a ser investigado é o preenchimento automático de uma parede isolada em sistema construtivo de alvenaria racionalizada de amarração 1/2 e 1/3. Dentre os componentes do sistema tem-se os blocos de concreto ou cerâmica de pertencente ao módulo de $15 \mathrm{~cm}$ (ver figura 01). Além dos componentes complementares como cintas, vergas, contravergas e vãos, que não serão investigados nesta pesquisa.

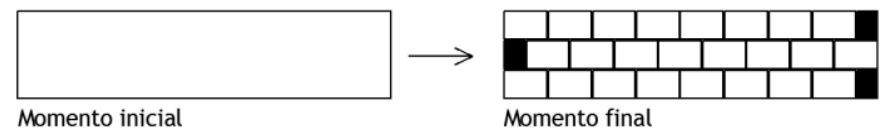

Figura 01: Comportamento paramétrico da alvenaria

\subsection{Fase de design}

Durante a fase de design foram observados restrições e parâmetros do sistema construtivos tendo como referência os principais comportamentos primitivos dos objetos elencados por Cavieres et al [14]: como fixação, rotação, translação e remodelação. Além das relações geométricas entre os objetos: alinhamento, igual espaçamento horizontal e restrição normal.

Quanto a rotação observa-se nos planos XZ, o sistema só permite a rotação a 90 graus da parede. Quanto aos planos YZ ou YZ, verifica-se que não é possível haver nenhum tipo de rotação, já que a parede não pode ser construída na horizontal (ver figura 02).
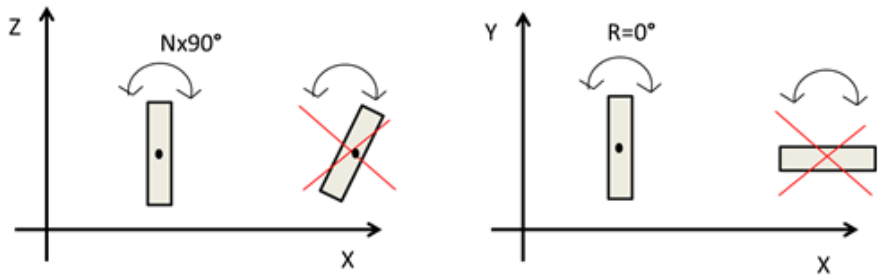

Figura 02: Restrições de rotação

Considerando a restrição de remodelação, ou seja, a transformação da forma ou das dimensões do objeto. As paredes 
e seus componentes devem obedecer às dimensões modulares do módulo $\mathrm{M}$, isto é, o módulo de $15 \mathrm{~cm}$ no sentido do plano XZ. Já na direção dos planos $\mathrm{YX}$ e $\mathrm{YZ}$, o módulo será $\mathrm{N}$, cujo o valor é $20 \mathrm{~cm}$. A utilização de dois módulos é possível, pois não há interferência das dimensões do eixo $\mathrm{Y}$ nos outros eixos, sendo desta forma necessária, pois é apenas possível encontrar no mercado componentes com dimensões modulares de $20 \mathrm{~cm}$ de altura, $15 \mathrm{~cm}$ de espessura e $15 \mathrm{~cm}$ de comprimento, sendo no caso os blocos de concreto e cerâmicos.

Os blocos que compõem as paredes são de 3 tipos: 3M, 2M, $1 \mathrm{M}$ com as respectivas dimensões modulares: $15 \mathrm{~cm} x 20 \mathrm{~cm} \times 45 \mathrm{~cm} ; 15 \mathrm{~cm} \times 20 \mathrm{~cm} \times 30 \mathrm{~cm}$ e $15 \mathrm{~cm} \times 20 \mathrm{~cm} x 15 \mathrm{~cm}$. Porém, de acordo com a amarração adotada no sistema, os mesmos podem ser trabalhados de formas distintas. No sistema de amarração $1 / 2$ os blocos de $2 \mathrm{M}$ são os blocos principais de preenchimento da paginação os blocos de $1 \mathrm{M}$ são blocos complementares e os blocos de $3 \mathrm{M}$ são blocos de amarração entre paredes. Já no sistema de amarração de 1/3, 3M é o principal e de amarração e os outros (1M e $2 \mathrm{M})$ blocos são complementares.

Como os blocos possuem dimensões fixas e estão inseridos nas paredes, os seus comportamentos de rotação e translação são iguais ao comportamento das paredes. Porém, a paginação destes blocos depende das dimensões das paredes e das relações de alinhamento, espaçamento horizontal, vertical e restrição normal entre os diferentes blocos. Os blocos de uma forma geral estão posicionados com suas aberturas voltadas para o chão, gerando uma relação normal. Quanto à relação entre os demais blocos, é mantido um alinhamento em relação à espessura e altura. Porém, no sentido do comprimento, o encontro entre eles possui um espaçamento igual de um $1 / 2$ ou $1 / 3$ dependendo do tipo de amarração de forma evitar juntas a prumo. Além de que todos os blocos são conectados por uma junta de argamassa de $1 \mathrm{~cm}$ (figura 03).

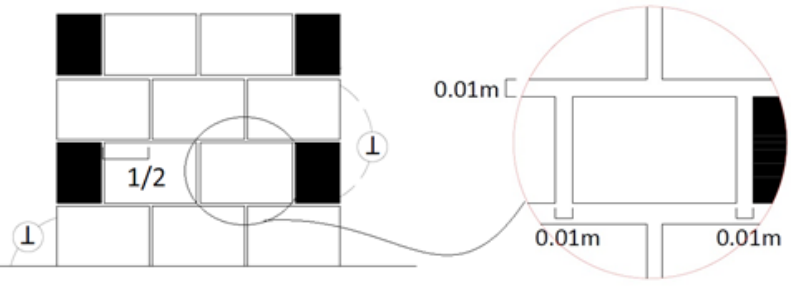

Figura 03: sistema de amarrações 1/2 de tijolos

Com a apresentação das restrições, deduziu-se que a espessura, por ser fixa, e a rotação das paredes, por não gerar nova distribuição dos blocos, não afetam no desenvolvimento da paginação. Logo, o desenho das paginações é influenciado somente pela altura, pelo comprimento e pela forma de amarração entre os blocos. Assim, para atender os requisitos acima, para a amarração $1 / 2$ se adotou a fórmula $C=n 2 M+R$, onde $\mathrm{C}$ representa as possíveis dimensões horizontais; $\mathrm{n}$, o número de blocos; $\mathrm{M}$, o valor do módulo horizontal e $\mathrm{R}$, o resto, que pode ser $1 \mathrm{M}$, ou zero. Quanto ao comportamento vertical temos uma fórmula similar ao horizontal, no caso, $\mathrm{H}=n 2 G+R$, onde $H$ representa todas as medidas verticais possíveis; $n$, o número de fiadas, $\mathrm{G}$, o valor do módulo vertical e o resto, que pode ser uma fiada ou nenhuma fiada. Com a descoberta dessas variáveis cogitou-se a existência de 04 padrões, vistos seguir (ver figura 05):

- C1H1 - quando o "R" da medida horizontal e vertical for igual a 01 ;

- $\mathrm{C} 2 \mathrm{H} 1$ - quando o "R" da medida horizontal for igual a 0 e o "R" da medida vertical for igual a 1 ;

- $\mathrm{C} 1 \mathrm{H} 2$ - quando o "R" da medida horizontal for igual a 01 e o " $\mathrm{R}$ " da medida vertical for igual a 0 ;

- $\mathrm{C} 2 \mathrm{H} 2$ - quando o " $\mathrm{R}$ " da medida horizontal e vertical for igual a 0 ;

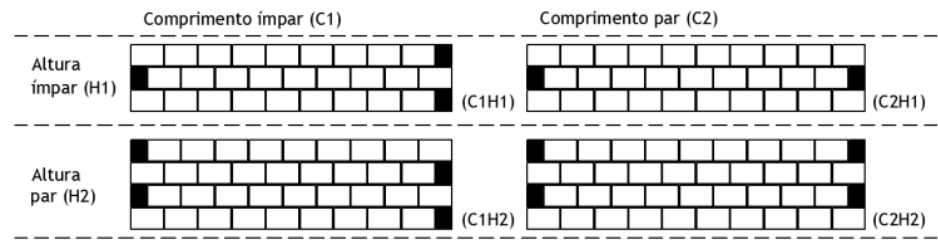

Figura 04: padrões previstos

Já para a amarração de $1 / 3$, todos os comprimentos devem obedecer a seguinte fórmula $\mathrm{C}=\mathrm{nx} 3 \mathrm{M}+\mathrm{R}$, onde $\mathrm{C}$ representa os possíveis comprimentos; $\mathrm{n}$, o número de blocos; $\mathrm{M}$, o valor do módulo horizontal e $\mathrm{R}$ o resto, que pode ser $1 \mathrm{M}, 2 \mathrm{M}$ ou zero. Quanto ao comportamento vertical temos uma fórmula similar ao horizontal, no caso, $\mathrm{H}=\mathrm{nx} 2 \mathrm{G}+\mathrm{R}$, onde $\mathrm{H}=$ representa todas as medidas verticais possíveis; $n$, o número de fiadas, G, o valor do módulo vertical e o resto que pode ser 01 fiada ou nenhuma fiada.

- R1H1 - quando o "R" da medida horizontal e vertical for igual a 01 ;

- $\quad \mathrm{R} 2 \mathrm{H} 1$ - quando o "R" da medida horizontal for igual a 2 e o "R" da medida vertical for igual a 1;

- R3H1 - quando o "R" da medida horizontal for igual a 0 e o "R" da medida vertical for igual a 01 ;

- $\mathrm{R} 1 \mathrm{H} 2$ - quando o "R" da medida horizontal for igual a 1 e o "R" da medida vertical for igual a 0 ;

- R2H1 - quando o "R" da medida horizontal for igual a 02 e o "R" da medida vertical for igual a 0 ;

- $\mathrm{R} 3 \mathrm{H} 2$ - quando o "R" da medida horizontal e vertical for igual a 0 ;

Assim, com a descoberta de existência de padrões de dimensões horizontais e verticais, geramos uma contribuição para trabalhos anteriores de Romcy (2012) e Vilató et Franco (2000) que apesar de apresentarem equações se baseiam somente em padrões horizontais. 


\subsection{Fase de Validação}

Durante esta etapa os padrões foram modelados e submetidos a testes dentro do software Archicad 18 em nível de script, onde foram simuladas diversas situações. No método BOB original as simulações foram desenvolvidas através de scripts.

Os modelos desenvolvidos possuem representações tridimensionais e bidimensionais que, no caso, foi optado somente pelas bidimensionais, pois permitem uma visualização mais objetiva e clara do comportamento dos padrões. Os experimentos consistiram em gerar variações na altura e no comprimento das paredes. Além de observar o comportamento de seus algoritmos para gerar as paginações. Assim, os padrões foram validados utilizando-se de testes que verificavam o comportamento paginação a partir da variação do comprimento e altura das paredes, baseados nos seus respectivos módulos. Logo, os testes foram agrupados em dois tipos de experimentos: um realizando variações de medidas horizontais (módulo M) e outro apresentando variações de medidas verticais.

Os testes de validação das medidas horizontais ocorreram da seguinte forma: primeiro foi utilizada uma parede qualquer com tamanho modular e realizou-se uma simulação em script do comportamento esperado, isto é preencheu-se toda a parede com os blocos de tijolos, observando-se as imagens geradas e anotando o comportamento. $\mathrm{O}$ teste foi descrito por meio de textos de imagens, descrevendo passo a passo do comportamento da paginação das paredes: desde o momento inicial, com a parede lisa até o momento final com a parede totalmente preenchida com os blocos (ver figura 05).

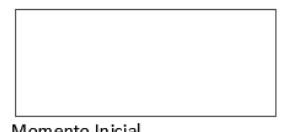

Momento Inicial

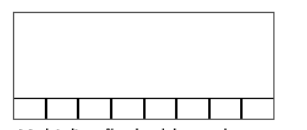

Multiplicação dos blocos de $2 \mathrm{M}$ da esquerda para direita.

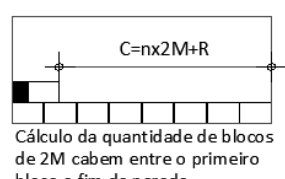

de $2 \mathrm{M}$ cabem entre o primeir

bloco e fim da parede.

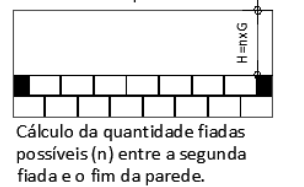

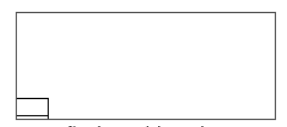

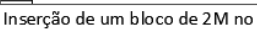
canto esquerdo da primeira fiada.
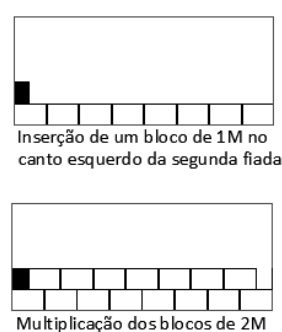

Multiplicação dos blocos de $2 \mathrm{M}$ da esquerda para direita.

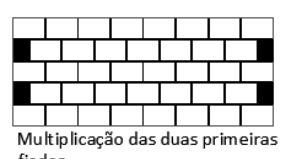
fiadas.

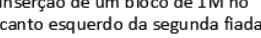

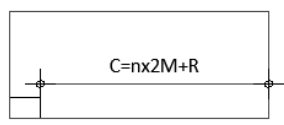

Cálculo da quantidade de blocos de $2 M(n)$ cabem entre $o$ primeiro bloco e fim da parede.
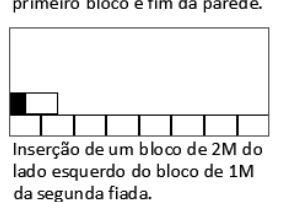
da segunda fiada.

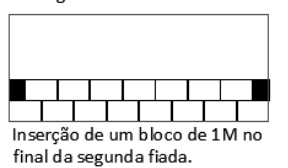

final da segunda fiada.

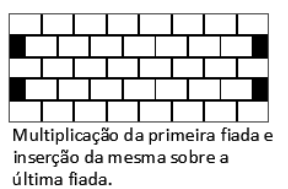
última fiada.

Figura 05: Simulação em script do tipo C1H1

Do mesmo modo ocorrem os testes sequenciais. Porém, com diferença que nos experimentos seguintes ampliou-se o

valor de um módulo, isto é, $15 \mathrm{~cm}$ da parede em relação a parede anterior. No total foram feitos 04 testes, onde se observou que o primeiro teste obteve-se, padrão $\mathrm{C} 1 \mathrm{H} 1$ e no seguinte o padrão $\mathrm{C} 2 \mathrm{H} 1$. Os testes 03 e 04 repetiram os padrões anteriores de forma alternada. Assim, conclui-se que, se continuássemos os testes, com números múltiplos $\mathrm{M}$, obteríamos os mesmos resultados, o que comprovou a existência única destes dois padrões. Quanto aos testes referentes às medidas verticais, foram realizados da mesma forma, porém, manteve-se o comprimento fixo e gerou-se variações modulares de $20 \mathrm{~cm}$ para cima (ver figura 06). Ao fim dos testes, todos os padrões previstos foram validados sem surgir nenhum novo padrão.

A validação dos testes para sistemas de $1 / 3$ foi similar, havendo também a validação dos padrões previstos (ver figura 07). Porém, tivemos que realizar 06 testes, no caso dos testes de dimensões horizontais, pois somente a cada três realizados se repetia a sequencia de padrões previstos para o comprimento. Já no caso dos testes verticais o número de testes foi múltiplo de 02 . Assim, o experimento anterior todos os padrão da amarração de $1 / 3$ foram validados.

1. Amarração $1 / 2$
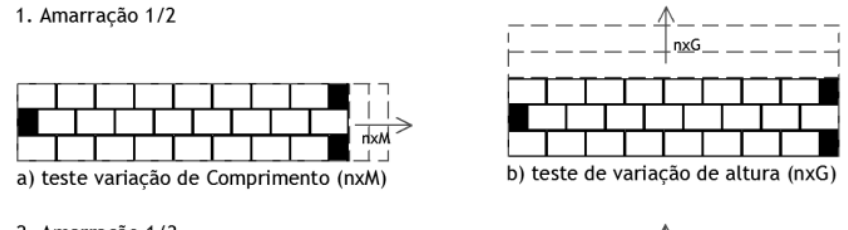

2. Amarração $1 / 3$
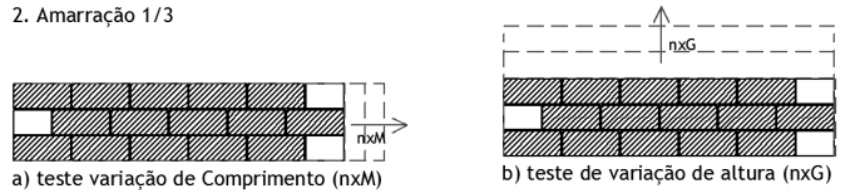

Figura 06: Testes de comprimento e altura das amarrações 1/2 e 1/3.

\subsection{Fase de tradução}

Após a validação dos padrões fora realizada a tradução de seus comportamentos para diagramas UML. O processo de tradução ocorreu em três etapas. Primeiro extraímos os fluxos de ações descritos em cada padrão dos conjuntos amarração $1 / 2 \mathrm{e}$ amarração 1/3 simulados em script. Depois os comparamos por tipo padrão padrões de amarração 1/2: C1H1, C2H1, C1H2 e padrões amarração 1/3: C2H2 e R1H1, R2H1, R3H1 R1H2, $\mathrm{R} 2 \mathrm{H} 2$ e R3H2. Por fim, construímos o diagrama.

Para cada conjunto de padrões foi gerado quadros comparativos a partir de dados extraídos, onde as ações de cada tipo de padrão foram inseridas em uma coluna distinta. Com o intuito de facilitar a identificação de ações similares, para cada padrão adotamos uma cor. Logo, quando as ações são iguais adotamos a mesma cor (ver tabelas 01 e $02 \mathrm{em}$ anexo). Assim, com a geração da tabela foi possível verificar os fluxos de ações convergentes e divergentes em cada padrão. 
Com finalização da tabela dos padrões de amarração de $1 / 2$, se observa variações de fluxos convergentes e divergentes, oque resulta em um diagrama composto de fluxo de ações contínuo, seguido elementos como bifurcações e barras de união. Outro ponto a ser observado é que, apesar de existirem quatro padrões, os fluxos só dividem em dois caminhos (ver figura 07).

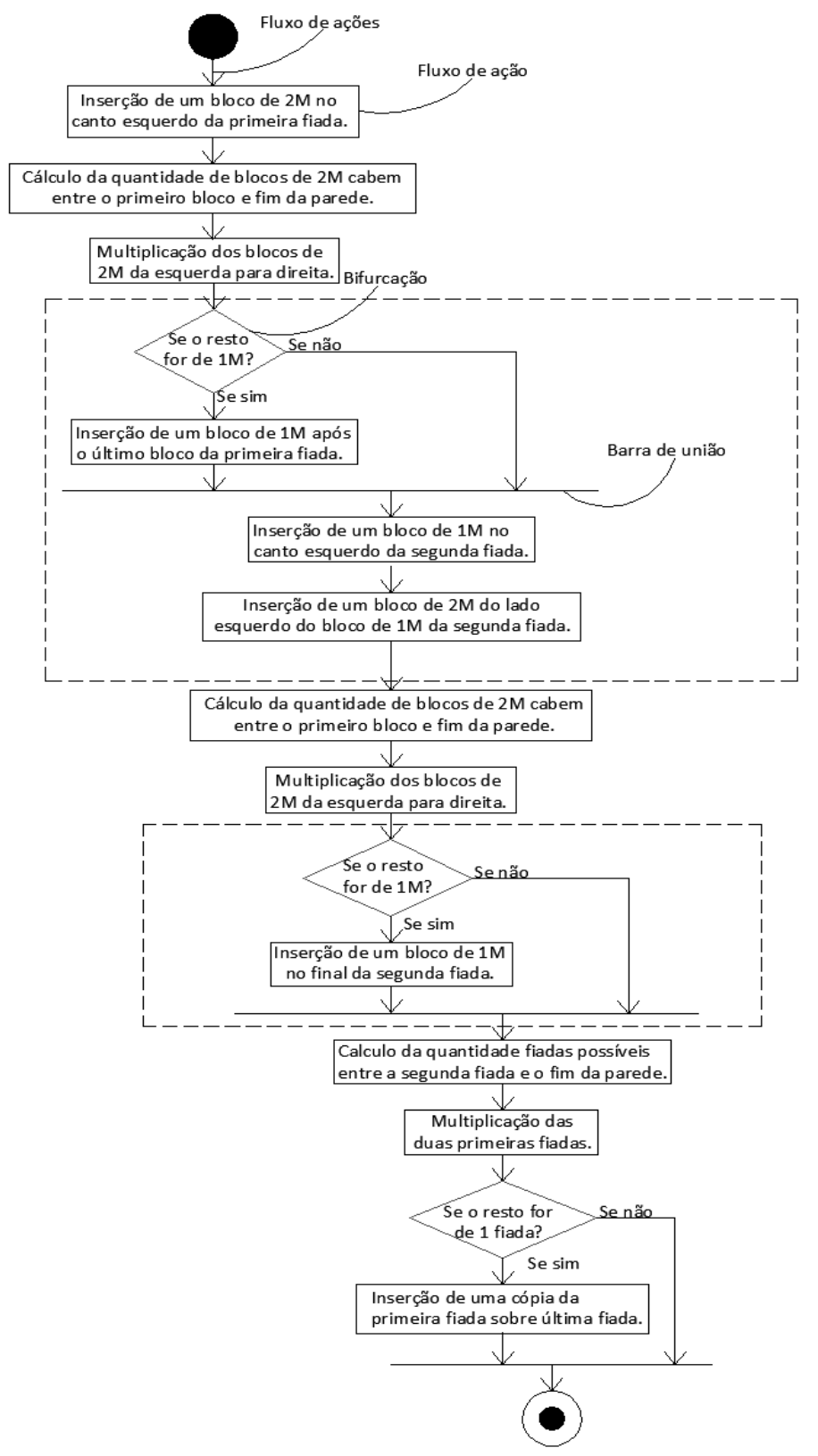

Figura 07 - Diagrama de atividades amarração 1/2

Observando-se como variam os fluxos de ações tendem convergir ou divergir na amarração $1 / 3$ (ver tabela 02 ), verificase a formação de um diagrama muito similar ao da amarração de
1/2. Porém, com pequenas diferenças: o bloco de $3 \mathrm{M}$ é o bloco principal e a presença de duas bifurcações interligadas, gerando 03 caminhos distintos (ver figura 08)

Com a criação dos diagramas, percebe-se que apesar das paredes possuírem amarrações distintas, apresentam ações comuns ou muitos similares e um formato bem similar com pequenas variações: sejam ações iguais onde varia apenas o bloco principal, invés de $2 \mathrm{M}$, utiliza-se o de $3 \mathrm{M}$ ou o surgimento de novas bifurcações para atender os padrões de $1 \mathrm{M}, 2 \mathrm{M}$ e $3 \mathrm{M}$.

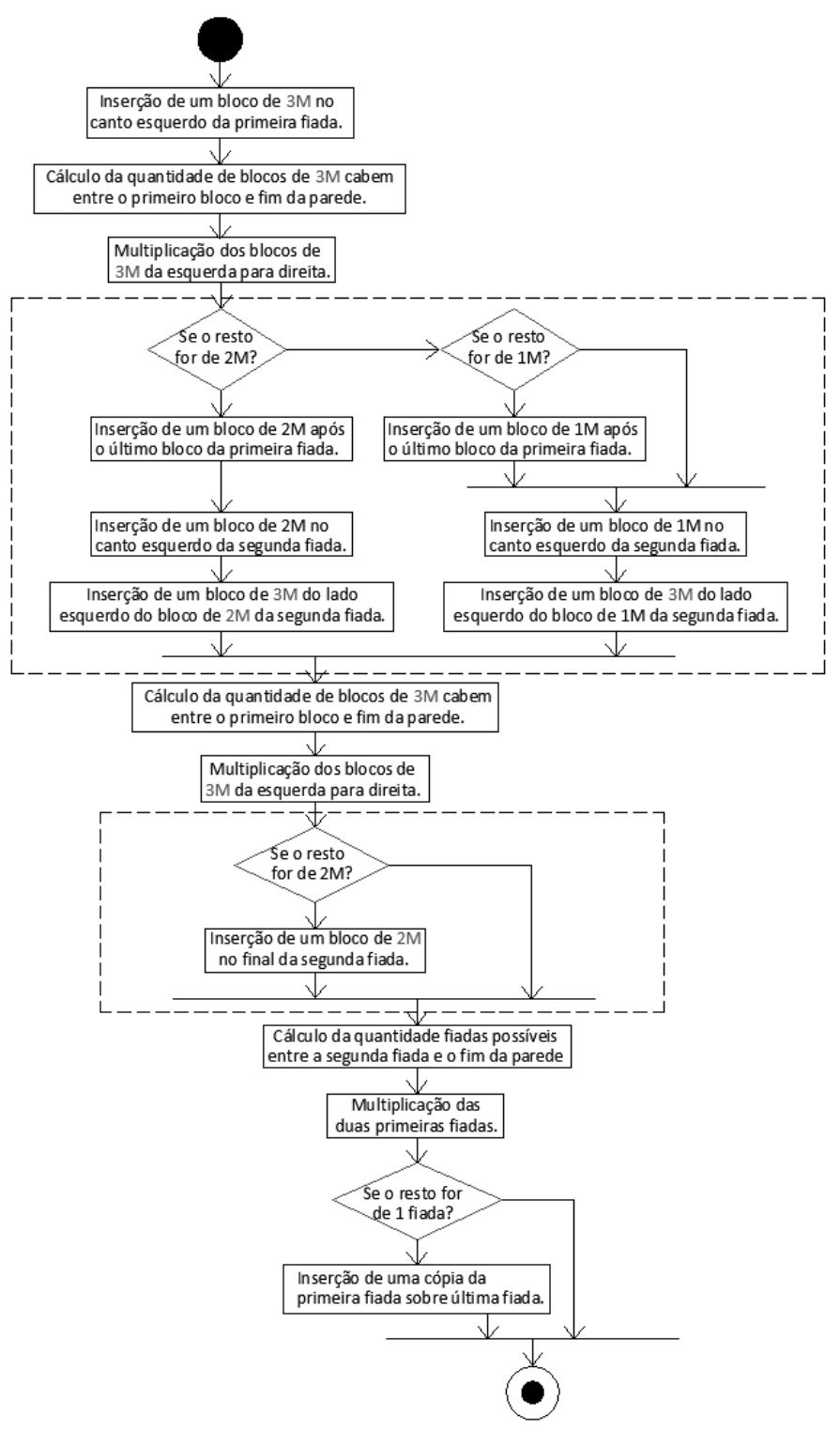

Figura 08 - Diagrama de atividades amarração 1/3 


\section{Conclusões}

Ao fim da pesquisa percebe-se que os objetivos da mesma foram atingidos de uma forma plena, pois foi possível identificar e validar os padrões de comportamento e gerar diagramas aplicáveis em plataformas BIM, onde de comprovarmos a eficiência do método $\mathrm{BOB}$ e da simulação em script.

Dentro dos padrões gerados percebe-se que objetos paramétricos de mesma natureza apresentam padrões de comportamento similares, quanto às equações paramétricas, padrões ação e reação e diagramas de atividades. Logo, supõe-se que a automação inserção novos componentes ou subsistemas, como vãos, paredes, lajes e instalações, sobre as paredes de amarração um $1 / 2$ e $1 / 3$ sempre apresentaram comportamento equivalentes. $\mathrm{O}$ que pode facilitar o desenvolvimento de diagramas e a automação de ferramentas para ambos os sistemas. $\mathrm{O}$ mesmo se imagina que a automação de peças de estruturais de concreto e peças com vigas e pilares, podem gerar comportamentos similares, em termos de forma.

Ao verificarmos o surgimento de novos padrões não previstos por Romcy [16] referente à altura das paredes, oque valida a teoria que modelagem paramétrica tem mais eficiência do que a gramática da forma no quesito de identificação dos padrões. Quesito já apontado por Cavieres et al [14]. Dentre as ferramentas que auxiliaram nesse processo destacam-se os comportamentos primitivos e as relações entre os componentes. Além da utilização da ferramenta simulação em script, que tornou mais clara a visualização dos resultados de execução dos algoritmos.

\section{Agradecimentos A FINEP}

\section{STUDIES FOR DESIGN AUTOMATION THE MASONRY RATIONALIZED IN BIM PLATFORMS}

\begin{abstract}
This research comes to inquiry ways of automating the masonry design in computer systems. Soon aims to develop applicable diagrams in BIM platforms, enabling the automatic generation of paging masonry rationalized into two types $1 / 2$ and $1 / 3$ mooring. The research strategy used was the research constructive, based for the notation method building object behavior (BOB) developed in the period 2001-2004 by North American researchers as Chang Lee, Rafael Sacks and Charles Eastman. The research is important, as contributing to the development of BIM platforms, specifically in the design phase, optimizing productivity and efficiency. After the research was possible to generate their development, still understand the degree of convergences and divergences between them in the category of computer diagrams.
\end{abstract}

Keywords: automation design, BIM, parametric modeling, masonry rationalized

\section{Referências}

[1] FOCHEZATTO, Adelar et GHINIS , Cristiano Ponzoni. Determinantes do crescimento da construção civil no Brasil e no Rio Grande do Sul: evidências da análise de dados em painel. Ensaios FEE, Porto Alegre, v. 31, Número Especial, p. $648-678$, jun. 2011

[2] ADDOR, Miriam Roux Azevedo; CASTANHO, Miriam Dardes de Almeida; CAMBIAGHI, Henrique; DELATORRE, Joyce Paula Martin; NARDELLI, Eduardo Sampaio; OLIVEIRA, André Lompreta. Colocando o "i" no BIM. Revista Acadêmica Arquitetura e Urbanismo, São Paulo, n.4, p.104-115, 2010

[3] MONTEIRO, A.; FERREIRA, R. C.; SANTOS, E. T. Algumas abordagens para representação detalhada de elementos de paredes de alvenaria em ferramentas BIM. In: TIC2009: ENCONTRO DE TECNOLOGIA DA INFORMAÇÃO E COMUNICAÇÃO NA CONSTRUÇÃO CIVIL, 4., 2009, Rio de Janeiro. Anais... Rio de Janeiro: ANTAC, 2009.

[4] MÜLLER, Ana Luiza. Informações para placas cerâmicas de revestimento segundo a abordagem BIM. 2010. 167f. Dissertação (Mestrado em Engenharia de Edificações e Saneamento) - Programa de Pós-graduação em Engenharia Civil pela Universidade Estadual de Londrina, Londrina/PR, 2010.

[5] SILVA, Margarete Maria de Araújo. Diretrizes para o projeto de alvenarias de vedação. 2003. 167 p. Dissertação (Mestrado em Engenharia) - Escola Politécnica, Universidade de São Paulo, São Paulo, 2003.

[6] FERREIRA, Rita Cristina ; SANTOS, Eduardo Toledo. Uma proposta de uso do CAD 3D em projetos para produção de vedações verticais em edifícios. São Paulo: EPUSP, 2008. 22p. (Boletim Técnico da Escola Politécnica da USP).

[7]SUCCAR, Bilal. Building information modelling framework: a research and d elivery foundation for industry stakeholders. [8] ARAYICI, Y, Coates, P, Koskela, LJ, Kagioglou, M, Usher, C and O'Reilly, K. Technology adoption in the BIM implementation for lean architectural practice. 2011. Automation Construction, n.20, p.189-195, jan. 2011

[9] SACKS, Rafael; EASTMAN, Charles M.; LEE, Ghang. Parametric 3D modeling in building construction with examples from precast concrete.

[10] AYRES FILHO, Cervantes. Acesso ao modelo integrado do edifício. Dissertação (Mestrado em Construção Civil) - Universidade Federal do Paraná, Curitiba, 2009.

[11] CHECCUCCI, E. S.; PEREIRA, A. P.; AMORIM, A. L. A difusão das tecnologias BIM por pesquisadores do Brasil. In: ENCONTRO DE TECNOLOGIA DE INFORMAÇÃO E COMUNICAÇÃO NA CONSTRUÇÃO, 5., 2011, Salvador. Anais... Salvador: UFBA, 2011. CD-ROM. Uma Visão da Difusão e Apropriação do Paradigma BIM no BRASIL - TIC 2011

[12] LEE, Ghang; SACKS, Rafael; EASTMAN, Charles M. Specifying parametric building object behavior (BOB) for a building information modeling system. Automation in Construction, v. 15, n. 6, p.758-776, nov. 2006.

[13] EASTMAN, Charles; TEICHOLZ, Paul; SACKS, Rafael et LISTON, Kathleen. BIM handbook: a guide to building information modeling for owners, managers, designers, engineers and contractors. Hoboken: Wiley, 2008, 490 p.

[14] CAVIERES, Andres. GENTRY, Russell; AL-HADDAD, Tristan. Knowledge-based parametric tools for concrete masonry walls: conceptual design and preliminary structural analysis. Automation in Construction, v.20, n.6, p.716728 , out. 2011.

[15] AYRES, C.; AZUMA, F. e SCHEER, S. Utilização de CAD BIM para projeto de alvenaria de blocos de concreto. In: Workshop Brasileiro de Gestão do Processo de Projetos na Construção de Edifícios, 8, 2008, São Paulo. 


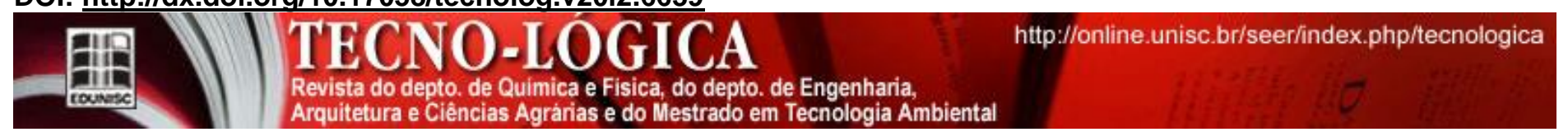

[16] ROMCY, Neliza Maria e Silva. Proposta de tradução dos princípios da coordenação modular em parâmetros aplicáveis ao building information modeling. 2012. 181f. Dissertação (Mestrado em Engenharia Civil) Universidade Federal do Ceará. Fortaleza, 2012.

[17] CELANI, Gabriela; CYPRIANO, Débora; GODOI, Giovana.; VAZ, Carlos Eduardo V. A gramática da forma como metodologia de análise e síntese em arquitetura. Conexão: comunicação e cultura, v.5, n.10, p.181-197, 2006.

[18] BETTING, B.; SHAH, J. Derivation of a standard set of geometric constraints for parametric modeling and data exchange. Computer-Aided Design, v.33, n.1, p.17-33, jan. 2000
[19] LUKKA, Kari. The constructive research approach. In: OJALA, L.; HILMOLA, O-P. (Eds.). Case study research in logistics. Turku: Turku School of Economics and Business Administration, 2003. p.83-101.

[20] VAISHNAVI , Vijay K.;KUECHLER JR., William. Design science research methods and patterns: innovating information and communication technology. Boston: Auerbach Publications,2007. 
ANEXOS

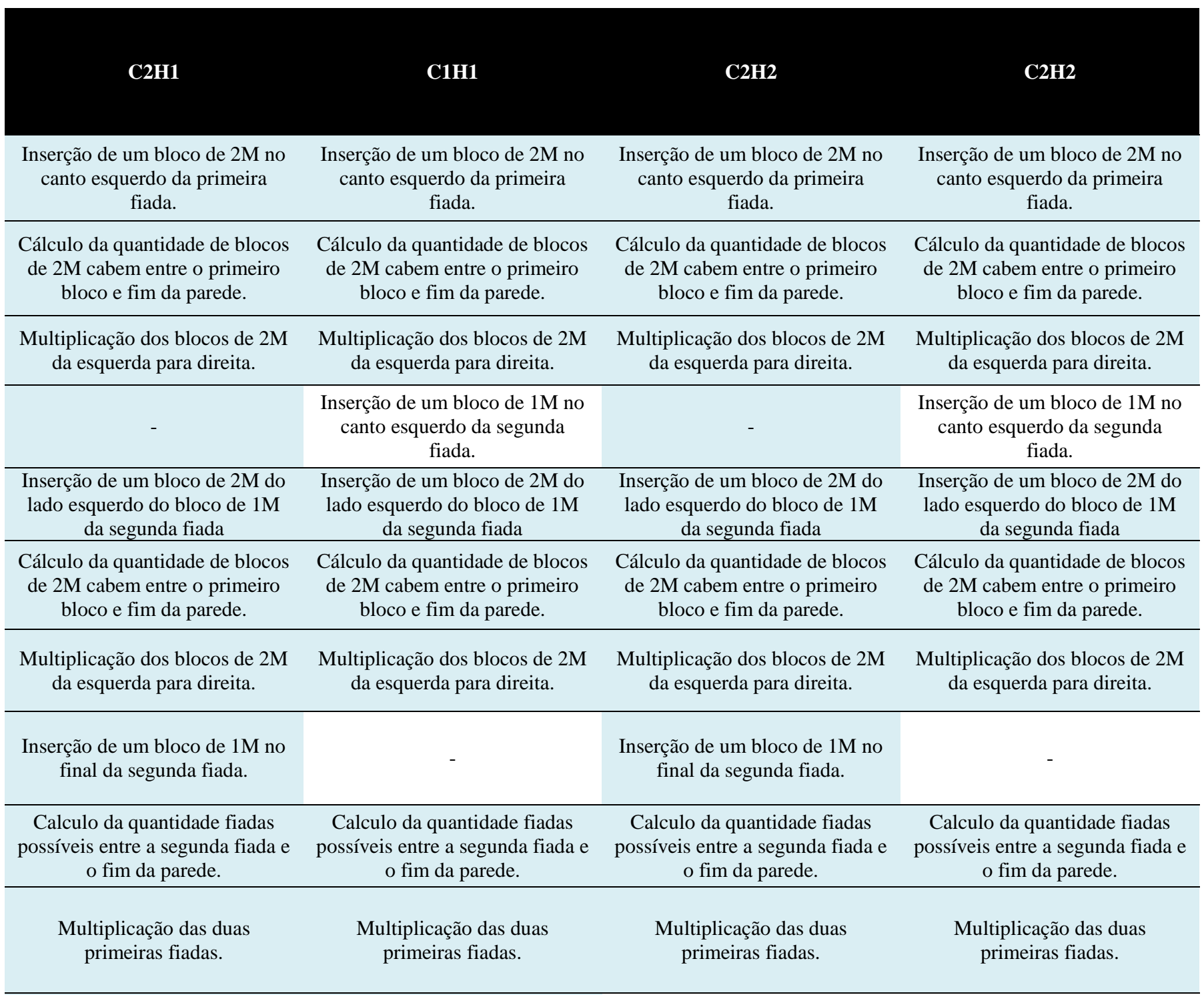

Inserção de uma cópia da primeira fiada sobre última fiada.
Inserção de uma cópia da primeira fiada sobre última fiada.

Tabela 01 - Análise comparativa dos padrões de amarração 1/2 


\begin{tabular}{|c|c|c|c|c|c|}
\hline R2H1 & R1H1 & R3H1 & $\mathrm{R} 2 \mathrm{H} 2$ & $\mathrm{R} 1 \mathrm{H} 2$ & R3H2 \\
\hline $\begin{array}{l}\text { Inserção de um bloco } \\
\text { de } 3 \mathrm{M} \text { no canto } \\
\text { esquerdo da primeira } \\
\text { fiada. }\end{array}$ & $\begin{array}{l}\text { Inserção de um bloco } \\
\text { de } 3 \mathrm{M} \text { no canto } \\
\text { esquerdo da primeira } \\
\text { fiada. }\end{array}$ & $\begin{array}{l}\text { Inserção de um bloco } \\
\text { de } 3 \mathrm{M} \text { no canto } \\
\text { esquerdo da primeira } \\
\text { fiada. }\end{array}$ & $\begin{array}{l}\text { Inserção de um bloco } \\
\text { de } 3 \mathrm{M} \text { no canto } \\
\text { esquerdo da primeira } \\
\text { fiada. }\end{array}$ & $\begin{array}{l}\text { Inserção de um bloco } \\
\text { de } 3 \mathrm{M} \text { no canto } \\
\text { esquerdo da primeira } \\
\text { fiada. }\end{array}$ & $\begin{array}{l}\text { Inserção de um bloco } \\
\text { de } 3 \mathrm{M} \text { no canto } \\
\text { esquerdo da primeira } \\
\text { fiada. }\end{array}$ \\
\hline $\begin{array}{l}\text { Cálculo da quantidade } \\
\text { de blocos de } 3 \mathrm{M} \\
\text { cabem entre o } \\
\text { primeiro bloco e fim } \\
\text { da parede. }\end{array}$ & $\begin{array}{l}\text { Cálculo da quantidade } \\
\text { de blocos de } 3 \mathrm{M} \\
\text { cabem entre o } \\
\text { primeiro bloco e fim } \\
\text { da parede. }\end{array}$ & $\begin{array}{l}\text { Cálculo da } \\
\text { quantidade de blocos } \\
\text { de } 3 \mathrm{M} \text { cabem entre o } \\
\text { primeiro bloco e fim } \\
\text { da parede. }\end{array}$ & $\begin{array}{l}\text { Cálculo da } \\
\text { quantidade de blocos } \\
\text { de } 3 \mathrm{M} \text { cabem entre o } \\
\text { primeiro bloco e fim } \\
\text { da parede. }\end{array}$ & $\begin{array}{l}\text { Cálculo da } \\
\text { quantidade de blocos } \\
\text { de } 3 \mathrm{M} \text { cabem entre o } \\
\text { primeiro bloco e fim } \\
\text { da parede. }\end{array}$ & $\begin{array}{l}\text { Cálculo da } \\
\text { quantidade de blocos } \\
\text { de } 3 \mathrm{M} \text { cabem entre o } \\
\text { primeiro bloco e fim } \\
\text { da parede. }\end{array}$ \\
\hline $\begin{array}{l}\text { Multiplicação dos } \\
\text { blocos de } 3 \mathrm{M} \text { da } \\
\text { esquerda para direita. }\end{array}$ & $\begin{array}{l}\text { Multiplicação dos } \\
\text { blocos de } 3 \mathrm{M} \text { da } \\
\text { esquerda para direita. }\end{array}$ & $\begin{array}{l}\text { Multiplicação dos } \\
\text { blocos de 3M da } \\
\text { esquerda para direita. }\end{array}$ & $\begin{array}{l}\text { Multiplicação dos } \\
\text { blocos de } 3 \mathrm{M} \text { da } \\
\text { esquerda para direita. }\end{array}$ & $\begin{array}{l}\text { Multiplicação dos } \\
\text { blocos de } 3 \mathrm{M} \text { da } \\
\text { esquerda para direita. }\end{array}$ & $\begin{array}{l}\text { Multiplicação dos } \\
\text { blocos de } 3 \mathrm{M} \text { da } \\
\text { esquerda para direita. }\end{array}$ \\
\hline $\begin{array}{l}\text { Inserção de um bloco } \\
\text { de } 2 \mathrm{M} \text { após o último } \\
\text { bloco da primeira } \\
\text { fiada. }\end{array}$ & $\begin{array}{l}\text { Inserção de um bloco } \\
\text { de } 1 \mathrm{M} \text { após o último } \\
\text { bloco da primeira } \\
\text { fiada. }\end{array}$ & & $\begin{array}{l}\text { Inserção de um bloco } \\
\text { de } 2 \mathrm{M} \text { após o último } \\
\text { bloco da primeira } \\
\text { fiada. }\end{array}$ & $\begin{array}{l}\text { Inserção de um bloco } \\
\text { de } 1 \mathrm{M} \text { após o último } \\
\text { bloco da primeira } \\
\text { fiada. }\end{array}$ & - \\
\hline $\begin{array}{l}\text { Inserção de um bloco } \\
\text { de } 2 \mathrm{M} \text { no canto } \\
\text { esquerdo da segunda } \\
\text { fiada. }\end{array}$ & $\begin{array}{l}\text { Inserção de um bloco } \\
\text { de } 1 \mathrm{M} \text { no canto } \\
\text { esquerdo da segunda } \\
\text { fiada. }\end{array}$ & $\begin{array}{l}\text { Inserção de um bloco } \\
\text { de } 1 \mathrm{M} \text { no canto } \\
\text { esquerdo da segunda } \\
\text { fiada. }\end{array}$ & $\begin{array}{l}\text { Inserção de um bloco } \\
\text { de } 2 \mathrm{M} \text { no canto } \\
\text { esquerdo da segunda } \\
\text { fiada. }\end{array}$ & $\begin{array}{l}\text { Inserção de um bloco } \\
\text { de } 1 \mathrm{M} \text { no canto } \\
\text { esquerdo da segunda } \\
\text { fiada. }\end{array}$ & $\begin{array}{l}\text { Inserção de um bloco } \\
\text { de } 1 \mathrm{M} \text { no canto } \\
\text { esquerdo da segunda } \\
\text { fiada. }\end{array}$ \\
\hline $\begin{array}{l}\text { Inserção de um bloco } \\
\text { de 3M do lado } \\
\text { esquerdo do bloco de } \\
\text { 2M da segunda fiada. }\end{array}$ & $\begin{array}{l}\text { Inserção de um bloco } \\
\text { de 3M do lado } \\
\text { esquerdo do bloco de } \\
\text { 1M da segunda fiada. }\end{array}$ & $\begin{array}{l}\text { Inserção de um bloco } \\
\text { de 3M do lado } \\
\text { esquerdo do bloco de } \\
\text { 1M da segunda fiada. }\end{array}$ & $\begin{array}{l}\text { Inserção de um bloco } \\
\text { de 3M do lado } \\
\text { esquerdo do bloco de } \\
\text { 2M da segunda fiada. }\end{array}$ & $\begin{array}{l}\text { Inserção de um bloco } \\
\text { de 3M do lado } \\
\text { esquerdo do bloco de } \\
\text { 1M da segunda fiada. }\end{array}$ & $\begin{array}{l}\text { Inserção de um bloco } \\
\text { de } 3 \mathrm{M} \text { do lado } \\
\text { esquerdo do bloco de } \\
\text { 1M da segunda fiada. }\end{array}$ \\
\hline $\begin{array}{l}\text { Cálculo da quantidade } \\
\text { de blocos de } 2 \mathrm{M} \text { que } \\
\text { cabem entre o } \\
\text { primeiro bloco e fim } \\
\text { da parede. }\end{array}$ & $\begin{array}{l}\text { Cálculo da quantidade } \\
\text { de blocos de } 2 \mathrm{M} \text { que } \\
\text { cabem entre o } \\
\text { primeiro bloco e fim } \\
\text { da parede. }\end{array}$ & $\begin{array}{l}\text { Cálculo da } \\
\text { quantidade de blocos } \\
\text { de } 2 \mathrm{M} \text { cabem entre o } \\
\text { primeiro bloco e fim } \\
\text { da parede. }\end{array}$ & $\begin{array}{l}\text { Cálculo da quantidade } \\
\text { de blocos de } 2 \mathrm{M} \text { que } \\
\text { cabem entre o } \\
\text { primeiro bloco e fim } \\
\text { da parede. }\end{array}$ & $\begin{array}{l}\text { Cálculo da quantidade } \\
\text { de blocos de } 2 \mathrm{M} \text { que } \\
\text { cabem entre o } \\
\text { primeiro bloco e fim } \\
\text { da parede. }\end{array}$ & $\begin{array}{l}\text { Cálculo da } \\
\text { quantidade de blocos } \\
\text { de } 2 \mathrm{M} \text { cabem entre o } \\
\text { primeiro bloco e fim } \\
\text { da parede. }\end{array}$ \\
\hline $\begin{array}{l}\text { Multiplicação dos } \\
\text { blocos de } 3 \mathrm{M} \text { da } \\
\text { esquerda para direita. }\end{array}$ & $\begin{array}{l}\text { Multiplicação dos } \\
\text { blocos de 3M da } \\
\text { esquerda para direita. }\end{array}$ & $\begin{array}{l}\text { Multiplicação dos } \\
\text { blocos de 3M da } \\
\text { esquerda para direita. }\end{array}$ & $\begin{array}{l}\text { Multiplicação dos } \\
\text { blocos de 3M da } \\
\text { esquerda para direita. }\end{array}$ & $\begin{array}{l}\text { Multiplicação dos } \\
\text { blocos de 3M da } \\
\text { esquerda para direita. }\end{array}$ & $\begin{array}{l}\text { Multiplicação dos } \\
\text { blocos de 3M da } \\
\text { esquerda para direita. }\end{array}$ \\
\hline- & - & $\begin{array}{l}\text { Inserção de um bloco } \\
\text { de } 2 \mathrm{M} \text { no final da } \\
\text { segunda fiada. }\end{array}$ & - & - & $\begin{array}{l}\text { Inserção de um bloco } \\
\text { de } 2 \mathrm{M} \text { no final da } \\
\text { segunda fiada. }\end{array}$ \\
\hline $\begin{array}{l}\text { Calculo da quantidade } \\
\text { fiadas possíveis entre } \\
\text { a segunda fiada e o } \\
\text { fim da parede. }\end{array}$ & $\begin{array}{l}\text { Calculo da quantidade } \\
\text { fiadas possíveis entre } \\
\text { a segunda fiada e o } \\
\text { fim da parede }\end{array}$ & $\begin{array}{l}\text { Calculo da } \\
\text { quantidade fiadas } \\
\text { possíveis entre a } \\
\text { segunda fiada e o fim } \\
\text { da parede. }\end{array}$ & $\begin{array}{l}\text { Calculo da quantidade } \\
\text { fiadas possíveis entre } \\
\text { a segunda fiada e o } \\
\text { fim da parede. }\end{array}$ & $\begin{array}{l}\text { Calculo da quantidade } \\
\text { fiadas possíveis entre } \\
\text { a segunda fiada e o } \\
\text { fim da parede }\end{array}$ & $\begin{array}{l}\text { Calculo da } \\
\text { quantidade fiadas } \\
\text { possíveis entre a } \\
\text { segunda fiada e o fim } \\
\text { da parede. }\end{array}$ \\
\hline $\begin{array}{l}\text { Multiplicação das } \\
\text { duas primeiras fiadas. }\end{array}$ & $\begin{array}{l}\text { Multiplicação das } \\
\text { duas primeiras fiadas. }\end{array}$ & $\begin{array}{l}\text { Multiplicação das } \\
\text { duas primeiras fiadas. }\end{array}$ & $\begin{array}{l}\text { Multiplicação das } \\
\text { duas primeiras fiadas. }\end{array}$ & $\begin{array}{l}\text { Multiplicação das } \\
\text { duas primeiras fiadas. }\end{array}$ & $\begin{array}{l}\text { Multiplicação das } \\
\text { duas primeiras fiadas. }\end{array}$ \\
\hline $\begin{array}{l}\text { Multiplicação da } \\
\text { primeira fiada e } \\
\text { inserção da mesma } \\
\text { sobre a última fiada. }\end{array}$ & $\begin{array}{l}\text { Multiplicação da } \\
\text { primeira fiada e } \\
\text { inserção da mesma } \\
\text { sobre a última fiada. }\end{array}$ & $\begin{array}{l}\text { Multiplicação da } \\
\text { primeira fiada e } \\
\text { inserção da mesma } \\
\text { sobre a última fiada. }\end{array}$ & - & - & - \\
\hline
\end{tabular}

Tabela 02 - Análise comparativa dos padrões de amarração 1/3 